\title{
Neu in der SÄZ-Redaktion
}

\section{Bruno Kesseli}

Dr. med. et lic. phil., Chefredaktor

Die SÄZ-Redaktion heisst mit Urs Brügger, Ursina Pally, Milo Puhan und Charlotte Schweizer vier neue Mitglieder in ihrem Kreis willkommen. Die Redaktion ist überzeugt, dass die Expertise der neuen Redaktionsmitglieder der Qualität der Zeitschrift und damit auch den Leserinnen und Lesern der SÄZ zugutekommen wird. Wir freuen uns auf die Zusammenarbeit.

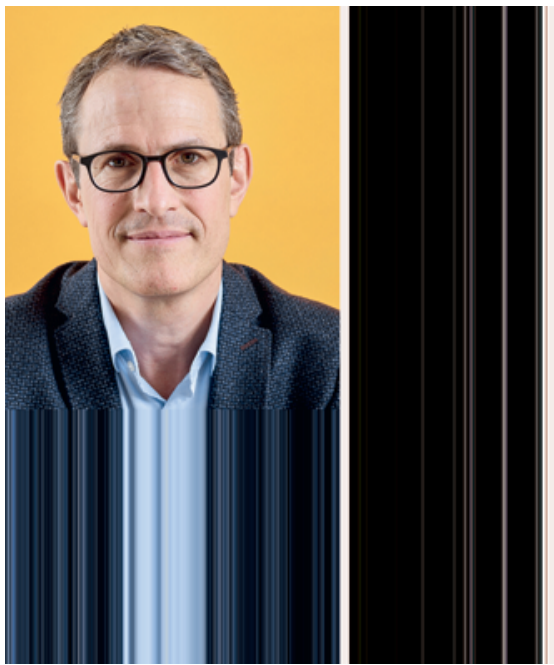

Urs Brügger studierte an der Universität St. Gallen Volkswirtschaftslehre und Soziologie. Er promovierte zum Dr. oec., verfügt über einen Master in Health Technology Assessment and Management und Zusatzausbildungen in Gesundheitsökonomie, Didaktik, Leadership und Coaching. Von 2003-2017 war er Leiter des Winterthurer Instituts für Gesundheitsökonomie an der Zürcher Hochschule für Angewandte Wissenschaften ZHAW. Seit Anfang 2018 leitet er als Direktor das Departement Gesundheit der Berner Fachhochschule BFH. Er ist seit 2013 Vorstandsmitglied und Quästor der Schweizerischen Akademie für Medizinische Wissenschaften SAMW. In der SÄZ-Redaktion tritt er die Nachfolge von Anna Sax an, die per Ende 2017 aus der Redaktion zurücktrat, weil sie 2018 die Leitung des Gesundheitsamts des Kantons Schaffhausen übernahm.

\section{Ursina Pally Hofmann}

Ursina Pally Hofmann leitet seit Juni dieses Jahres den Rechtsdienst der FMH. Nach der Erstausbildung zur Hebamme und rund 10-jähriger praktischer Berufserfahrung studierte sie an der Universität Zürich Rechtswissenschaften, promovierte zum Dr. iur., erwarb das Rechtsanwaltspatent und ergänzte ihre Ausbildung um ein "CAS Gesundheitssysteme» der Universitäten Zürich, Basel und Bern. Sie war während rund zehn Jahren als Rechtsanwältin in verschiedenen Bereichen tätig, unter anderem bei der FMH als stellvertretende Leiterin des Rechtsdienstes, in leitenden Funktionen bei einer grossen Versicherung sowie als Hochschuldozentin und Handelsrichterin. Die beiden letztgenannten Tätigkeiten übt sie im Nebenamt weiterhin aus. Bei der SÄZ übernimmt sie als Fachredaktorin Recht die Nachfolge von Hanspeter Kuhn.

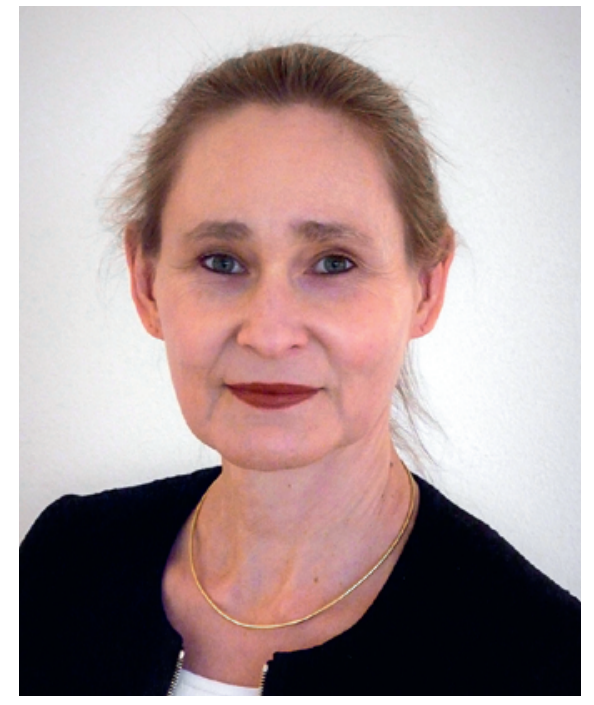


Milo Puhan

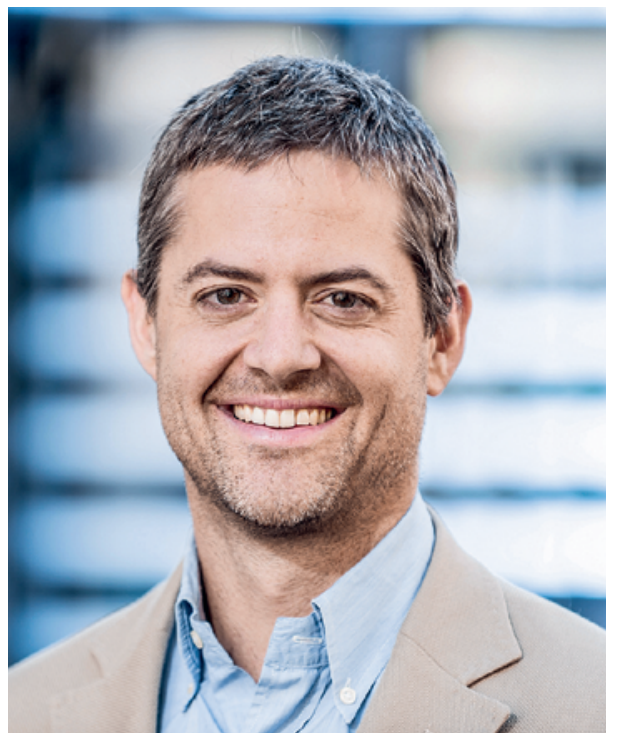

Milo Puhan studierte an der Universität Zürich Medizin und arbeitete danach zunächst als Assistenzarzt und später als Research Fellow im Horten-Zentrum der UZH. Seine Promotion in Epidemiologie erfolgte an der Universität Amsterdam. Von 2008 bis 2012 war er Associate Professor mit Tenure Track am Department of Epidemiology der Johns Hopkins Bloomberg School of Public Health. Seit 2013 ist er Ordinarius für Epidemiologie und Public Health an der Universität Zürich und Direktor des dortigen Instituts für Epidemiologie, Biostatistik und Prävention. Milo Puhans Hauptinteresse in der Forschung liegt auf der Prävention und dem Management von chronischen Krankheiten und der Entwicklung von Tools, welche eine Präferenzenbasierte Gesundheitsversorgung unterstützen. In der SÄZ-Redaktion ist Milo Puhan als Fachredaktor für den Bereich «Public Health, Epidemiologie und Biostatistik» zuständig.

\section{Charlotte Schweizer}

Charlotte Schweizer leitet seit Februar dieses Jahres die Abteilung Kommunikation der FMH. Nach ihrem Studium der englischen und französischen Sprach- und Literaturwissenschaften an der Universität Basel arbeitete sie in verschiedenen Funktionen für die CoopGruppe, darunter zuletzt als Projektleiterin in der Medienstelle. Danach war sie als Mediensprecherin beim Schweizerischen Komitee für UNICEF, dem Kinderhilfswerk der Vereinten Nationen, tätig. Berufsbegleitend absolvierte sie Studien der Kommunikations- und Medienwissenschaften an der Höheren Wirtschaftsschule Zürich (HWZ) und an der Journalistenschule MAZ in Luzern. Bei der SÄZ ist sie Mitglied des externen Redaktionsteams und übernimmt damit die Nachfolge von Jacqueline Wettstein.

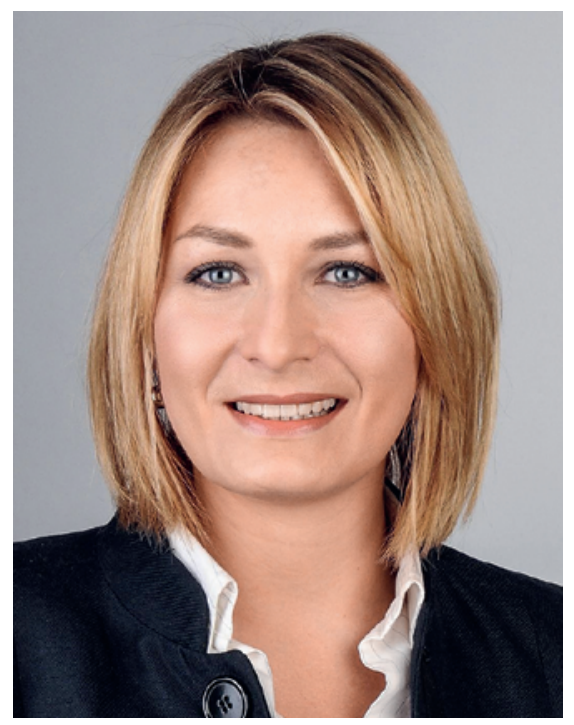

